Original Research Paper

\title{
Modulation of Aging in Yeast Saccharomyces cerevisiae by Roselle Petal Extract (Hibiscus sabdariffa L.)
}

\author{
Sarima, Rika Indri Astuti* and Anja Meryandini \\ Department of Biology, Faculty of Mathematics and Natural Sciences, \\ Bogor Agricultural University, Darmaga Campus, Bogor 16680, Indonesia
}

\author{
Article history \\ Received: 30-07-2018 \\ Revised: $20-08-2018$ \\ Accepted: 01-04-2019 \\ *Corresponding Author: \\ Rika Indri Astuti \\ Department of Biology, Faculty \\ of Mathematics and Natural \\ Sciences, Bogor Agricultural \\ University, Darmaga Campus, \\ Bogor 16680, Indonesia \\ Email: rikaindriastuti@apps.ipb.ac.id
}

\begin{abstract}
Cellular aging drives diseases. Free radicals are called the main cause of cellular aging. Antioxidants can protect the body from free radical attack by reducing its negative effects. An antioxidant source can be obtained from plants, such as roselle (Hibiscus sabdariffa. L). This research was conducted to analyze the ability of roselle petals extract to increase the life span of model organisms Saccharomyces cerevisiae BY4741. The $\mathrm{IC}_{50}$ value of extract-derived antioxidant activity based on DPPH and ABTS assays were $367,6 \mathrm{ppm}$ and 921,4 ppm, respectively. Antioxidant and antiaging tests were performed in vivo via spot assay. The result showed that the roselle petals extract-treated groups had the ability to extend the life span of yeast BY4741 under normal and oxidative stress conditions. This is also supported by the mitochondrial activity of BY4741 with roselle petals extract-treated group higher than those without the extract treatment. The $300 \mathrm{ppm}$ concentration was chosen as the best concentration of roselle petals extract to increase the life span in yeast. Interestingly, important gene to regulate aging in yeast (SIR2) as well as oxidative stress response genes (GPXI and GLR1) were significantly up-regulated in yeast cells treated with $300 \mathrm{ppm}$ roselle extract, as revealed via Real-time quantitative analysis. These results suggest that roselle petals extract exhibits antiaging effects likely via regulation of anti-oxidative stress and aging genes.
\end{abstract}

Keywords: Antioxidant, Antiglycation, Antiaging, Hibiscus sabdariffa. L, Saccharomyces cerevisiae

\section{Introduction}

Aging defines as a decline in physiological functions gradually, thereby reducing the growth rate (Young, 1997). Hayflick (1976) asserted that the lifespan of cells is limited. In normal condition, a cellular division occurred until reaching a limit, called as cellular aging. However, it comes earlier with presence of cellular distractors. In instance, accumulation of Reactive Oxygen Species (ROS) is reported to the factors that promote cellular aging (Gladyshev, 2014). As reported, cellular aging is responsible for a rising risk of some degenerative diseases such as Alzheimer, Parkinson and cancer (Niccoli and Linda, 2012).

ROS can be generated through normal metabolic process in human body, but in excessive level, it may induce oxidative stress. As free radicals, ROS had the ability to invade major molecules inside the cell such as DNA, lipid and protein (Dat et al., 2000). Various approaches been done to combat cellular aging. Among them, application antioxidant agents is considered as one of the promising way to reduce deleterious effect of ROS and AGEs molecules.

Currently, roselle (Hibiscus sabdariffa L.) tea drink has been believed to have positive effect to the human body. Indeed, some people are urged to consume the particular tea drink in daily basis. It is reported that roselle petals contain plenty of chemicals including water, protein, fat, fiber, calcium, phosphorous, iron, $\beta$ carotene, thiamine, riboflavin, niacin, ascorbic acid, vitamins (B1, B2 and D), anthocyanine, flavonoid and polyphenol (Liu et al., 2002; Lin et al., 2003; Zuraida et al., 2015). Several studies have showed that extracts of roselle could prevent diseases like cancer, obesity and cardiovascular diseases (atherosclerosis and coronary heart disease) (Tseng et al., 2000; Ochani and D'Mello, 2009; Alarcon-Aguilar et al., 2007; Carvajal-Zarrabal et al., 2009). Several groups of compounds in the extract, such as 
anthocyanins and protocatechuic acid, have been implicated as responsible for these effects (Da-Costa-Rocha et al., 2014). Hibiscus extract also demonstrated antibacterial, antifungal, antiparasitic, anti-inflammatory and antioxidant effect (Al-Hashimi, 2012; Mardiah et al., 2015). Protocatechuic acid compound in extract inhibited the growth of methicillin-resistant Staphylococcus aureus, Klebsiella pneumoniae, Pseudomonas aeruginosa and Acinetobacter baumannii (Liu et al., 2005). Moreover, roselle extract showed therapeutic promise in decreasing and preventing the development of atherosclerosis and possible related cardiovascular pathologies linked with diabetes (Da-Costa-Rocha et al., 2014). Although chemical properties of roselle are known, yet, the effects of roselle to cellular systems remain elusive. Indeed, their anti-aging activity has remained a great opportunity for investigation.

In this study, Saccharomyces cerevisiae BY4741 was used as model to evaluate the effects of roselle petal extract on aging pathways. Aging process in yeasts is complex and involves many cellular components conserved to that in mammalian systems. SIR2 gene is found to be one of the key factors related to the cellular aging. The gene is conserved in both yeasts and higher organisms and enables to regulate the aging processes such as regulation of stress defense, metabolism and glucose tolerance (Roux et al., 2010). The higher activity of SIR2 would prolong yeast's lifespan (Lin et al., 2016). Furthermore, mitochondrial activities are also involved in the cellular aging process. Strong mitochondrial membrane potential is also associated with extension of yeast's lifespan (Baracca et al., 2003). Indeed, high mitochondrial activity may induce mitochondrial-ROS adaptive signaling which culminate in the up-regulation of genes encoding cellular antioxidant enzymes such as glutathione peroxidase $(G P X 1)$ and glutathione reductase (GLR1), superoxide dismutase (SOD1) and catalase (CTT1). Their enzymatic activities are essential in scavenging ROS reactivity within cells, leading to extension of cell's lifespan (Bai et al., 2016).

This present work aimed to observe the antiaging activity of roselle petal extract applied in yeast $S$. cerevisiae as model and investigate its mechanism on how the aging process was delayed. Interestingly, we found that roselle extract is a potential antiaging agent that increases mitochondrial activity as well as gene involved in oxidative stress in yeast thus prolong yeast life span.

\section{Materials and Methods}

\section{Cultures and Medium}

The medium for culture of $S$. cerevisiae BY4741 was YPD (yeast extract peptone dextrose) containing 2\% glucose, $1 \%$ yeast extract, $2 \%$ peptone and $2 \%$ agar (Jarolim et al., 2004). Yeast in the room temperature was sub-cultured in a liquid YPD medium for stock culture and working culture, then incubated at room temperature $\left(25-28^{\circ} \mathrm{C}\right)$.

\section{Extraction of Roselle Petal}

The roselle petals were collected from Roselle Plantation in Madiun, East Java, Indonesia. The procedure for extraction was adopted from previous method (Zuraida et al., 2015). The roselle petals were macerated using ethanol $70 \%$ at ratio of $1: 5$ (sample:solvent). Sample was soaked for $3 \times 24 \mathrm{~h}$ and stirred twice a day to dissolve bioactive compounds in the sample. After macerated substance was obtained, the process was repeated twice using the same volume of solvent. The substance was collected and concentrated using rotary evaporator $\left(<60^{\circ} \mathrm{C}\right)$ to produce crude extract of roselle petals. The ethanol-derived extract of roselle petals showed the following characteristics: Paste, brownish red in color.

\section{Antioxidant Activity using DPPH and ABTS Assays}

The 1,1-diphenil-2-pikrilhidrazil (DPPH) procedure was conducted as described by previous study (Salazar et al., 2009). Briefly, extract was dissolved in absolute ethanol and the solution was made in 5 concentrations, including $150 \mathrm{ppm}, 300 \mathrm{ppm}, 450 \mathrm{ppm}$, $600 \mathrm{ppm}$ and $750 \mathrm{ppm}$. Sample $(100 \mu \mathrm{L})$ from each solution was transferred into a 96-well microplate (triplicates for each sample), added with $100 \mu \mathrm{l}$ of 125 $\mu \mathrm{M}$ DPPH in ethanol solutions and incubated at $37^{\circ} \mathrm{C}$ for $30 \mathrm{~min}$. The absorbance was measured at $514 \mathrm{~nm}$ using spectrophotometer.

The 2,2-azinobis-(3-ethylbenzothiazoline-6-sulfonic acid) (ABTS) procedure was performed according to previous study (Re et al., 1999). Briefly, ABTS solution was oxidized with $\mathrm{K}_{2} \mathrm{~S}_{2} \mathrm{O}_{8}$ to produce radicals. ABTS radicals $(180 \mu \mathrm{L})$ were transferred into 96-well microplate and added with sample solution $(20 \mu \mathrm{L})$ at triplicate. After incubation at dark room $\left(25-28^{\circ} \mathrm{C}\right)$ for $30 \mathrm{~min}$, the absorbance was measured using spectrophotometer at $734 \mathrm{~nm}$. Ascorbic acid was used as positive control. The $\mathrm{IC}_{50}$, defined as the concentration of substance that generates $50 \%$ reduction of free radicals, was determined. Percent inhibition was calculated using the following formula:

$$
\text { Inhibition }(\%)=1-\left(\frac{\text { Abs sample }- \text { Abs control }}{\text { Abs blank }- \text { Abscontrol }}\right) \times 100 \%
$$

Where:

Abs sample $=$ Absorbance of sample (the extracts + DPPH/ABTS)

Abs blank = Ethanol $+\mathrm{DPPH} / \mathrm{ABTS}$ Abs control is ethanol 
Table 1: Primer pairs for Real Time PCR used in this study

\begin{tabular}{lll}
\hline Gene & Forward primer & Reverse primer \\
SIR2 & CTTCCACTATGCCCGTACTGT & CGACATTGAACCCTGTGATGC \\
GPX1 & CAGTAAGCGGGAAGTCTGGAA & ACCACCTTCCCATTTCGGTC \\
ACT1 & CTATTGCAGCGGGCAGAAAG & GCTGGGGACGTTCTCGTAAT \\
\hline
\end{tabular}

\section{Aging Assay}

Aging assay was performed by using spot test, as described previously (Stephan et al., 2013). BY4741 was cultured in $3 \mathrm{ml}$ of liquid YPD for $24 \mathrm{~h}$ started at Optical Density (OD) of 0.1 . Assay was performed in five different treatments $(150,300,450,600$ and $750 \mathrm{ppm})$. Yeast culture without extract supplementation was used as negative control, while yeast cultured in low glucose $(0.5 \%)$ was used as positive control. A low glucose treatment is considered as Calorie Restriction (CR) conditions. Such CR conditions will induce yeast cellular mechanisms including mitochondrial activities respons to oxidative stress and autophagy, which contribute to the yeast's lifespan extension (Kaeberlein, 2010). Each culture was incubated for 15 days where spot test was conducted at each 10 and 15 days of incubations, Each culture was adjusted to $\mathrm{OD}=1$ then serially diluted (up to 10-4). About $2 \mu \mathrm{L}$ from each dilution suspension was then spotted on solid YPD medium. Spotted medium were incubated for 3 days at room temperature.

\section{Oxidative Stress Tolerance Assay}

The spot assay was carried out as above mentioned. Yet, about $2 \mu \mathrm{L}$ from each dilution suspension was spotted on solid YPD medium containing $\mathrm{H}_{2} \mathrm{O}_{2}$-induced oxidative stress in various concentrations $(7,8$ and $9 \mathrm{mM})$.

\section{Active Mitochondrial Staining}

The procedure was performed according to the previous study (Marchi and Cavalieri, 2008). Yeast BY4741 was incubated in liquid YPD medium supplemented with roselle petal extracts $(150,300,450,600$ and $750 \mathrm{ppm})$. The yeast suspension $(1 \mathrm{~mL})$ was centrifuged and the pellet obtained was suspended using phosphate buffer and added with 100 $\mathrm{nM}$ rhodamine B. Yeast suspensions were then incubated for $30 \mathrm{~min}$ at $25^{\circ} \mathrm{C}$. Light exposure was avoided throughout experiment. Mitochondrial activity was observed under fluorescence microscope (Olympus BX51).

\section{Gene Expression Assay using Real Time- Polymerase Chain Reaction (RT-PCR)}

Yeast BY4741 was treated with a selected concentration of roselle petal extract (300 ppm). Yeast was cultured in YPD medium and incubated in a shaking incubator for overnight at $30^{\circ} \mathrm{C}$. Positive and negative controls treatments were prepared as previously described in aging assay. Furthermore, mRNA extraction was conducted using RNeasy Mini Kit (Qiagen, USA). The total mRNA was used as template for cDNA synthesis using iSript cDNA Synthesis Kit (Bio-Rad, USA). RT-PCR experiment was performed using the particular cDNA template with Thunderbird SYBR qPCR Mix (Toyobo, Japan). RT-PCR analysis was conducted to investigate the effects of roselle petal extract on the expression of that aging-related gene n, i.e., SIR2 gene and response genes to the oxidative stress (glutathione peroxidase GPX1, glutathione reductase GLR1), using specific primers to each respective genes (Table 1). Data obtained from RT-PCR were analyzed using relative quantification method $2^{-\Delta \Delta \mathrm{CT}}$ (Livak and Schmittgen, 2001) and normalized towards reference genes $A C T 1$.

\section{Results and Discussion}

\section{Antioxidant Activities}

Antioxidant activity resulted from DPPH and ABTS assay was represented as $\mathrm{IC}_{50}$, resulting in value of 367.6 ppm and $921.5 \mathrm{ppm}$, respectively (Fig. 1A, Table 2). The $\mathrm{IC}_{50}$ for positive control (ascorbic acid) was much lower than that roselle petal extract (Fig. 1B and 1C). Antioxidant of the extract was low since it is a crude extract or not purified. It is worth noting that the roselle extract inhibited the activity of radical DPPH and ABTS in dose dependent manner (Fig. 1A). Compared to other plant extracts, $\mathrm{IC}_{50}$ of roselle petal extract (based on DPPH assay) was higher than citronella extract (Cymbopogon citratus), i.e., 1998 ppm (Lu et al., 2014). Meanwhile, $\mathrm{IC}_{50}$ of roselle petal extract (ABTS assay) was lower than flower extract of Helichrysum obconicum, i.e., 687.9 ppm (Figueira et al., 2014). Our results indicated that $\mathrm{IC}_{50}$ value based on DPPH and ABTS assays was dissimilar. It is due to different radicals used, which might elicit diverse responses of the antioxidative compounds. Similarly, the discrepancy on $\mathrm{IC}_{50}$ between DPPH and ABTS assay was also reported by former studies that investigated antioxidant activity of 50 foods (Floegel et al., 2011). This confirmed that each sample could exert various mechanisms on scavenging free radicals.

Based on in vitro study of antioxidant activities, the crude extract of roselle petals showed the highest antioxidant activity on scavenging DPPH radicals. This indicates that the extract is promising as antioxidant agent. Furthermore, we investigated antiaging activity of the extract by evaluating the lifespan extension of yeast S. cerevisiae BY4741. 


\section{Yeast Lifespan Analysis}

The result showed that Roselle petal extract (150, 300 and $450 \mathrm{ppm}$ ) could extend yeast's lifespan (Fig. 2) as observed in the effects of extracts on viability of yeast, compared with that without extract addition. Presence of the extract could produce better viability for 15-days culture in comparison with absence of the extract. The viability was even similar to the culture treated in CR conditions (positive control). In our experiment, administration of Roselle petal extract could extend the lifespan of yeast even though in nonCR condition, i.e., culture containing $2 \%$ glucose. This finding indicates that the extract may mimic regulation mechanism on the physiological system to that treated with $\mathrm{CR}$ condition, leading to lifespan extension.

To our knowledge, this is the first experiment to show the potential properties of Roselle petals as antiaging agent. Previously, a study on antiaging properties of herb plant Gastrodia elata (Orchidaceae) was reported (Lin et al., 2016). The proposed mechanism of antiaging properties is the improved cellular activity on neutralizing oxidative stress. Therefore, further experiment is carried out, focusing mainly on how the extract would induces oxidative stress tolerance mechanisms in yeast.

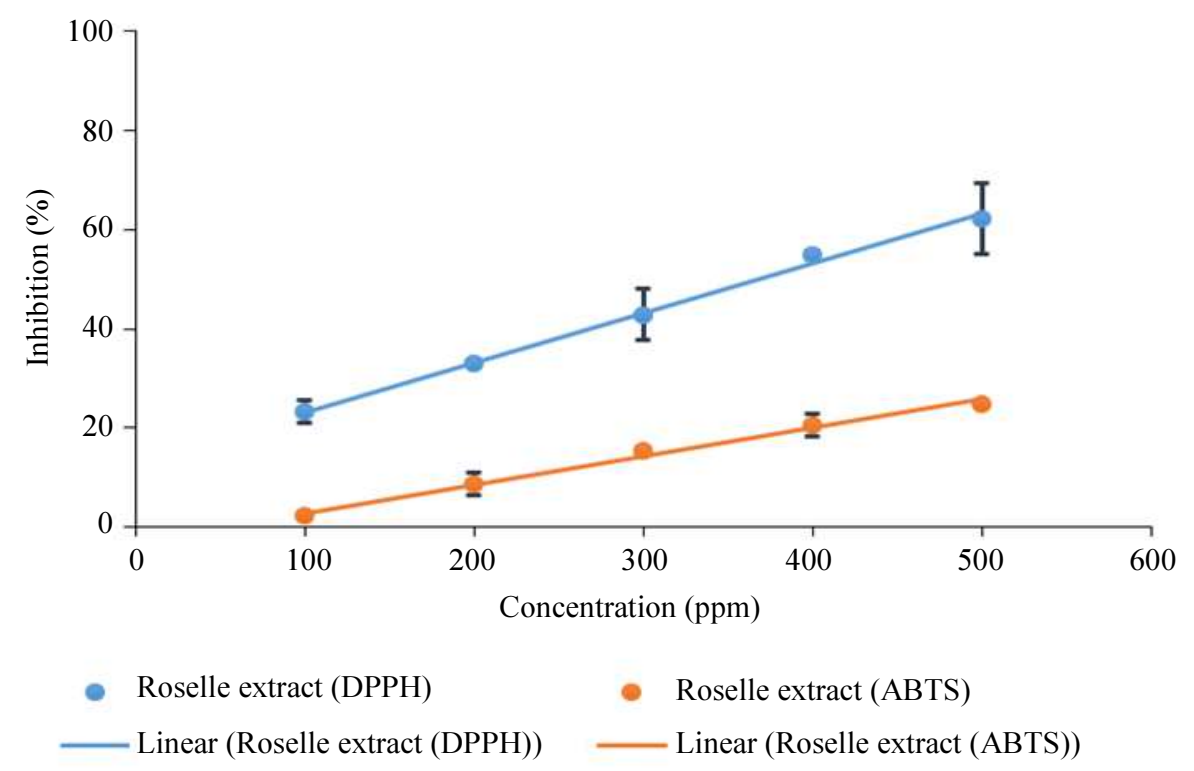

(A)

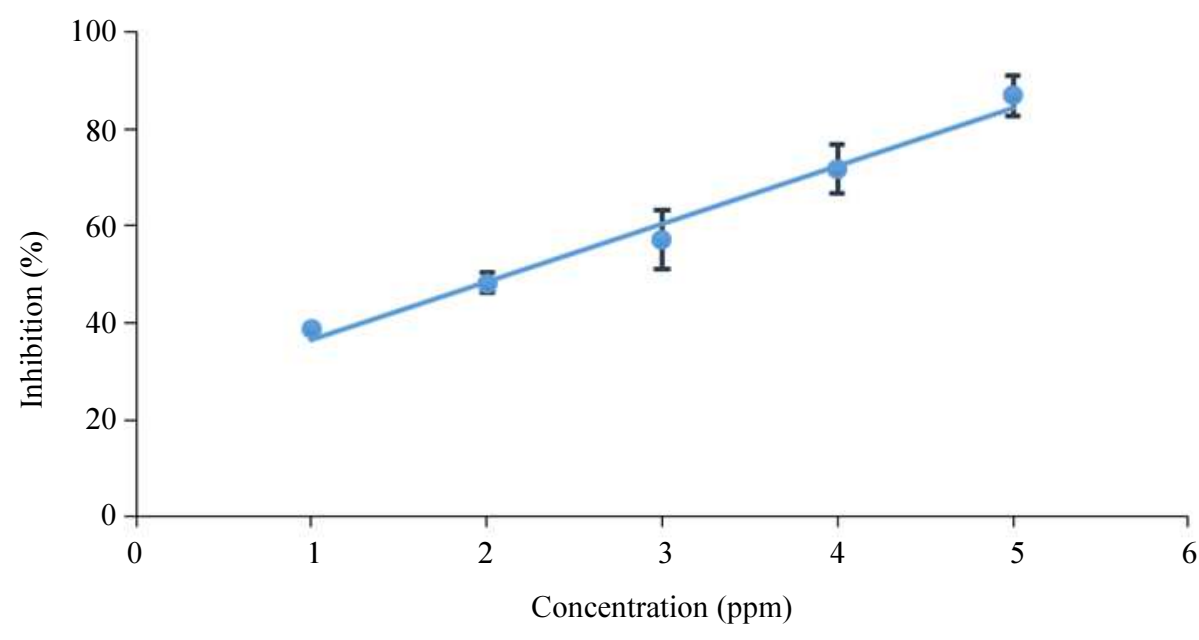

Ascorbic acid (DPPH) Linear (Ascorbic acid (DPPH)) 


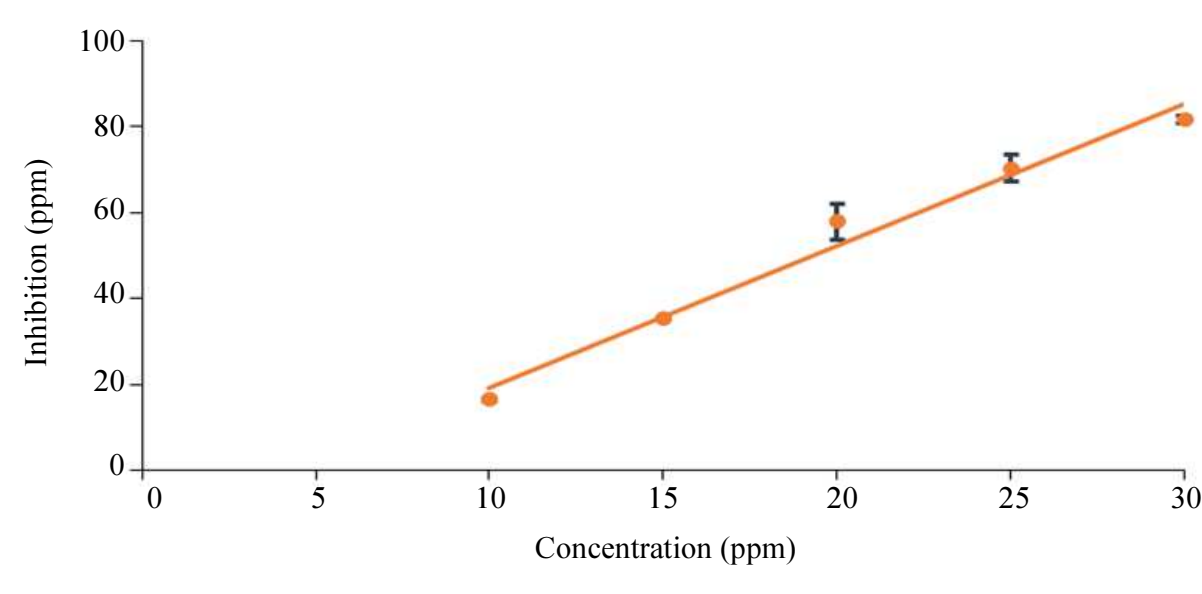

- Ascorbic acid (ABTS)

- Linear (Ascorbic acid (ABTS))

(C)

Fig. 1: Anti-oxidant activity of ethanol-derived roselle petal extract is lower than that control, ascorbic acid. (A) The anti-oxidant activity of Roselle petal extract is occurred in dose dependent manner against radical DPPH $\left(\mathrm{R}^{2}=0.996\right)$ and $A B T S\left(\mathrm{R}^{2}=\right.$ 0.991). Anti-oxidant activity of control substance, ascorbic acid, against (B) DPPH and (C) ABTS
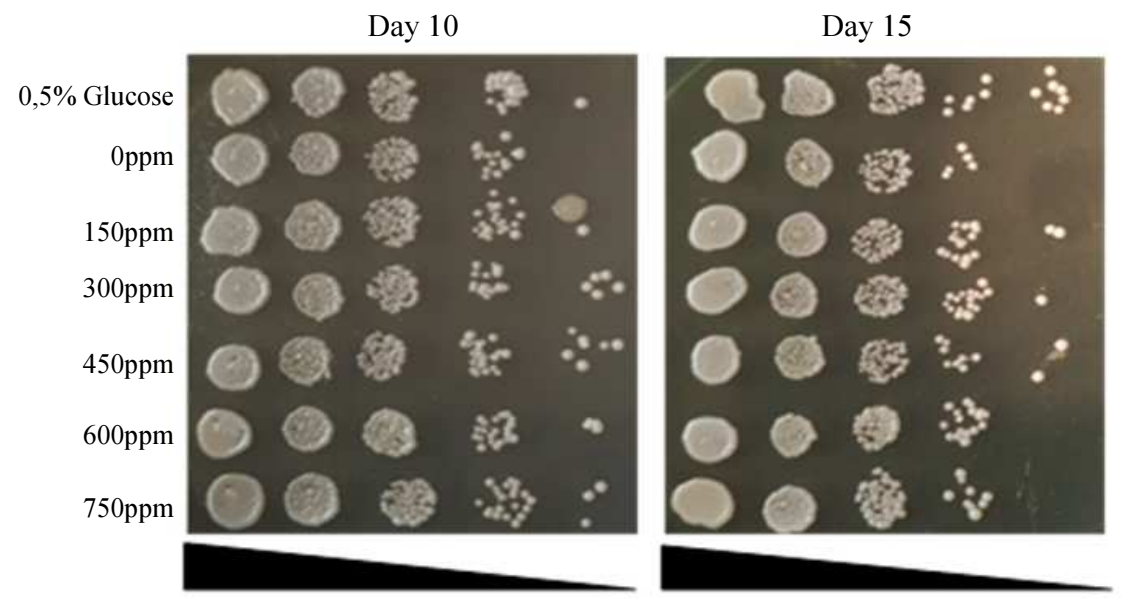

Fig. 2: Effects of Roselle petal extracts on the lifespan of $S$. cerevisiae BY4741. Yeast cells were grown in various concentrations of extract for 15 days. Spot test was performed at indicated time. Yeast cultured in YPD medium with low glucose $(0.5 \%)$ was used as positive control. Spotted medium were incubated for 3 days in room temperature

Table 2: Antioxidant and antiglycation activity of roselle petal extracts in vitro

\begin{tabular}{lll}
\hline & $\mathrm{IC}_{50}$ Antioxidant activity (ppm) \\
\hline Sample & DPPH assay & ABTS assay \\
\hline Ascorbic acid & $2.1 \pm 0.345^{\mathrm{a}}$ & $19.3 \pm 2.200^{\mathrm{a}}$ \\
Roselle petal extract & $367.6 \pm 5.214^{\mathrm{b}}$ & $921.4 \pm 13,400^{\mathrm{b}}$
\end{tabular}

Each value with different letters are significantly different within each assay at the level of $\mathrm{p}<0.05$

\section{Oxidative Stress Tolerance}

The results showed that treatment of roselle petal extract improved tolerance of yeast to $\mathrm{H}_{2} \mathrm{O}_{2}$-induced oxidative stress induced. This effect obviously occurred primarily on 15-days old yeast culture (Fig. 3).
Interestingly, the yeast viability treated with the extract was higher than that positive control (CR condition). In addition, we found that the extract at 300 ppm was regarded as the best concentration in relation to provide the most desirable viability against oxidative stress with a high concentration of $\mathrm{H}_{2} \mathrm{O}_{2}(9 \mathrm{mM})$. The capability of the extract on inducing intracellular oxidative stress responses for yeast could indicate promising candidate as pro-oxidant.

Compound with pro-oxidant activity enables to enhance cellular oxidation that involves free radicals. Previous study successfully reported polyphenols as the pro-oxidant agent isolated from green tea, capable of inducing oxidative stress responses in yeast through producing free radicals $\mathrm{H}_{2} \mathrm{O}_{2}$ (Maeta et al., 2007). The 
induction of intracellular response against oxidative stress is reported to be associated with mitochondrial activity. The rising mitochondrial activity allows to induce mitochondrial-ROS adaptive signaling mechanism which plays a central role in assisting yeast cells to cope with oxidative stress. The mechanism could be triggered through CR condition (Burtner et al., 2009). Hence, further test was performed to observe role of the extracts on mitochondrial activity.

\section{Mitochondrial Activity Assay}

The test aimed to observe the possible in vivo induction of yeast mitochondrial activity by roselle extract. In comparison with negative control (0 ppm), the mitochondrial activity of extract-treated yeast was much higher, as indicated by stronger and brighter red color (Fig. 4). Interestingly, the extract could induce mitochondrial activity in a dose-independent manner. As compared to positive control $(0.5 \%$ glucose $)$, mitochondrial activity of yeast treated with the extract seemed to be similar, even higher at extract concentration of $300 \mathrm{ppm}$. Potential energy in mitochondrial membrane could induce fluorescence from rhodamine, while the rate of fluorescence was comparable with potential membrane activity of mitochondria (Baracca et al., 2003). This suggests that brighter color means a greater mitochondrial activity. Our result showed that the addition of extract could enhance mitochondrial activity, in which the best effect was attributed to concentration of $300 \mathrm{ppm}$. Our finding confirmed that the roselle petal extract could likely promote mitochondria-ROS adaptive signalling mimicking to that intracellular stress response under CR condition. Additionally, Gastrodia elata was also reported to have similar effect (Lin et al., 2016).
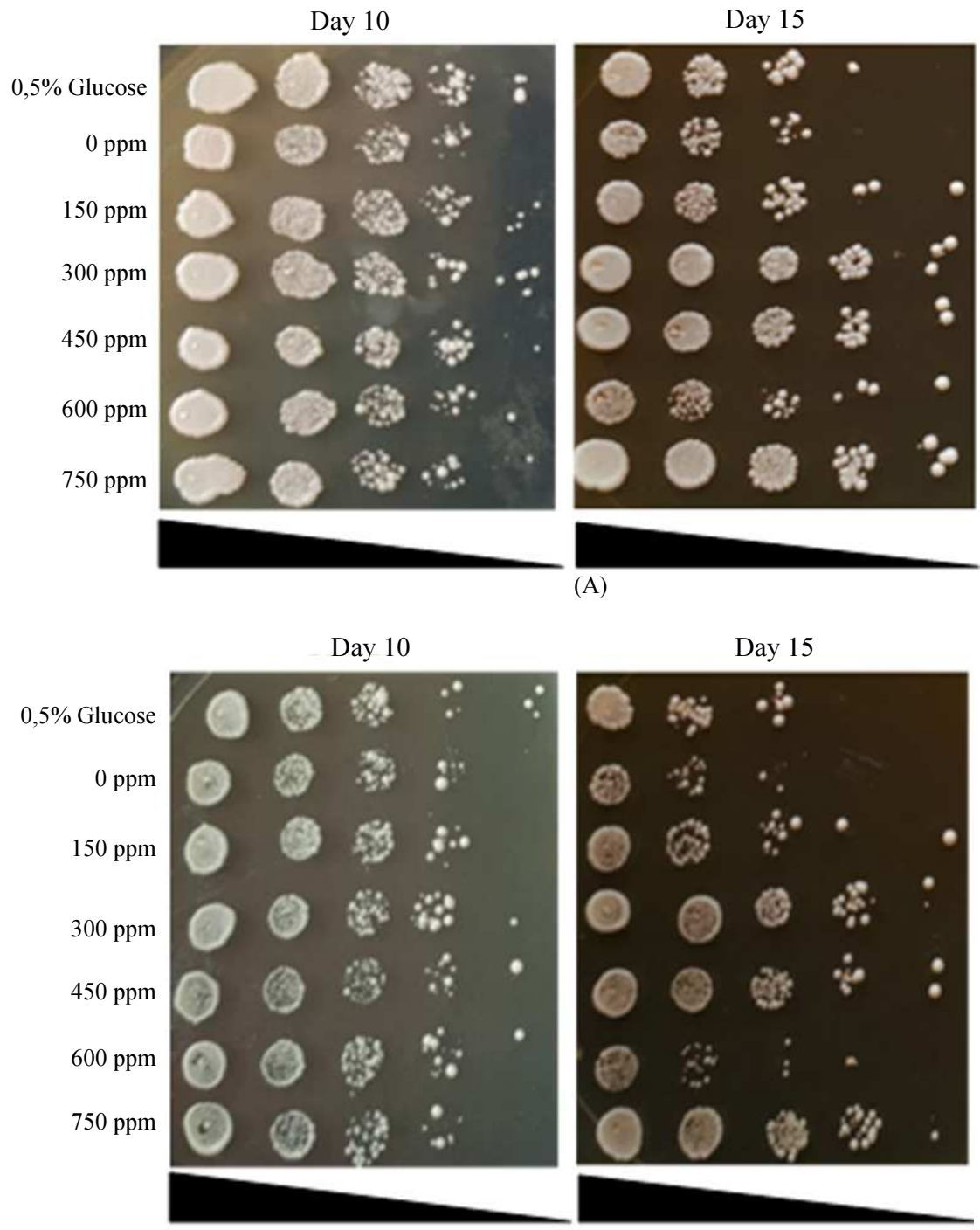

(B) 


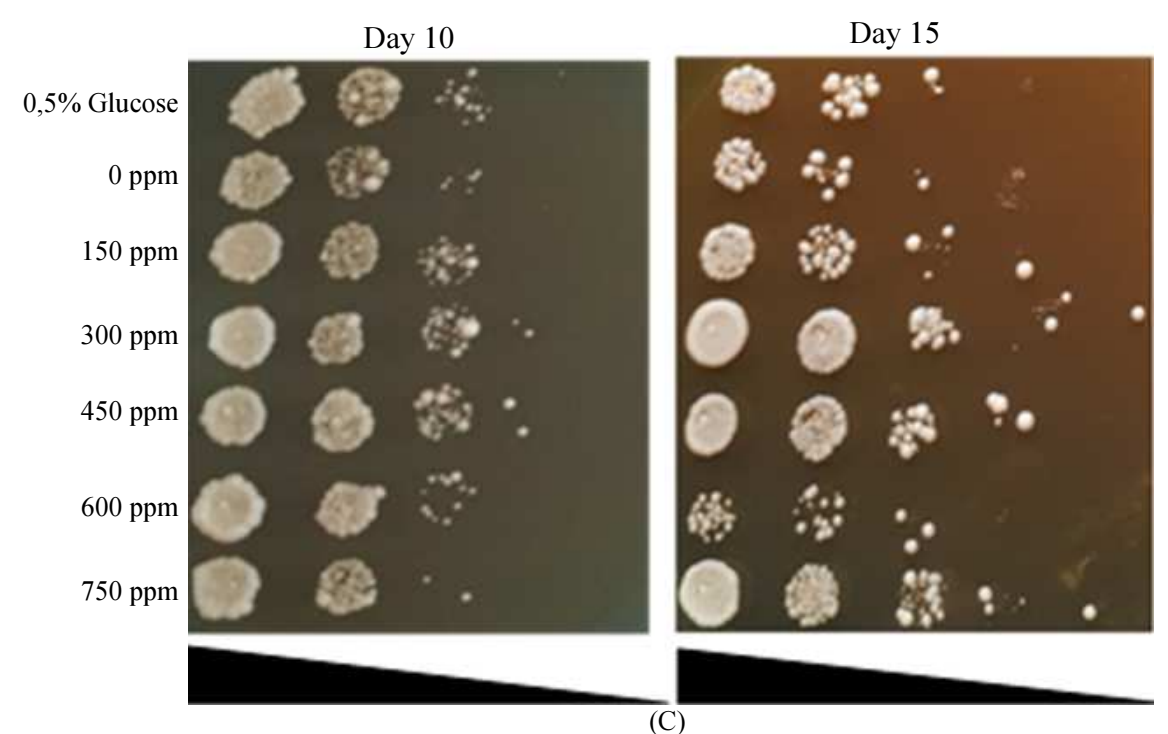

Fig. 3: Effects of Roselle petal extracts on viability of $S$. cerevisiae BY4741 against H2O2 at (A) $7 \mathrm{mM}$, (B) $8 \mathrm{mM}$ and (C) $9 \mathrm{mM}$. Yeast were grown in various concentrations of extract for 15 days. Spot test was performed at indicated time. Yeast cultured in YPD medium with low glucose $(0.5 \%)$ was used as positive control. Spotted medium were incubated for 3 days in room temperature
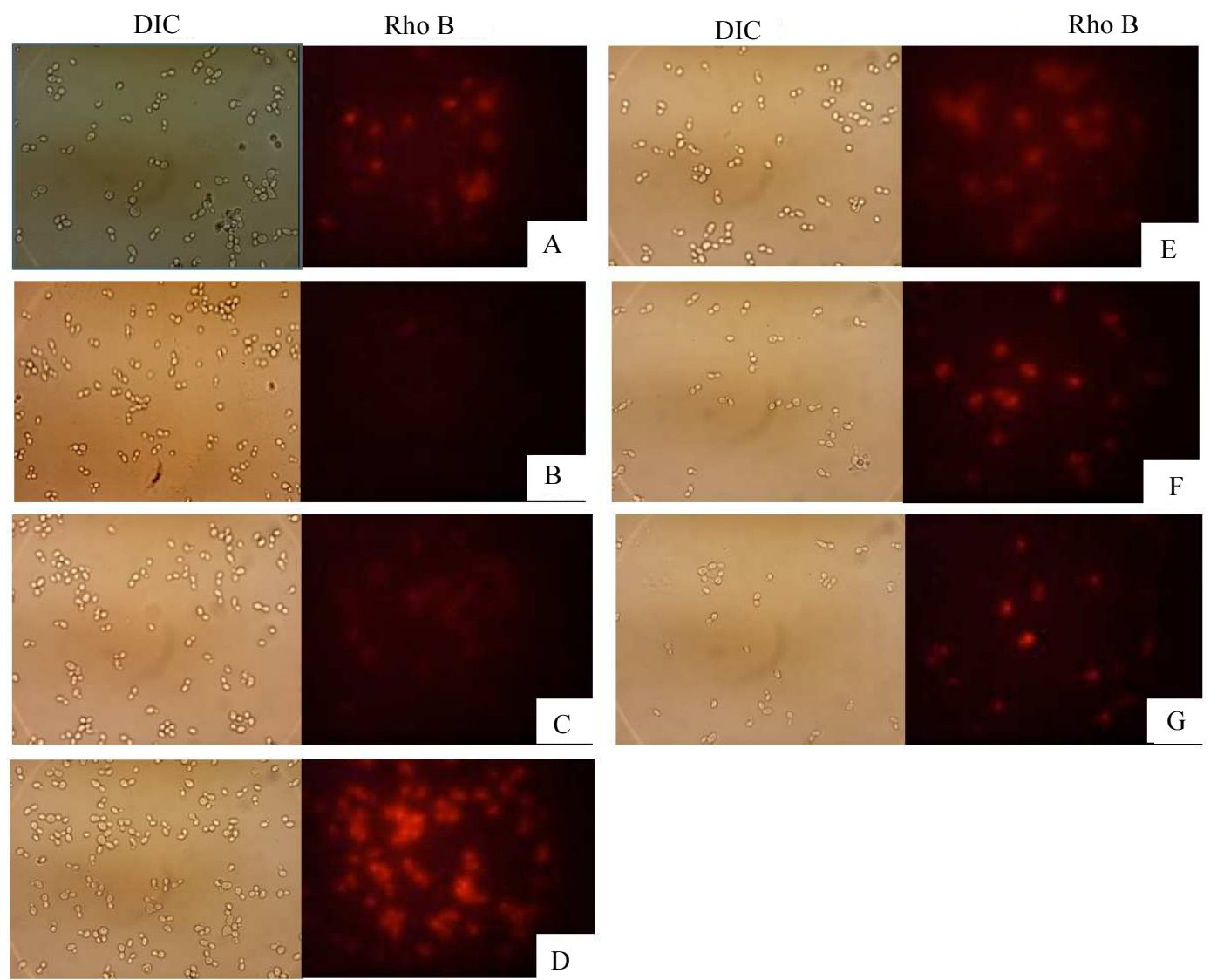

Fig. 4: The effects of Roselle petal extract on mitochondrial activity of $S$. cerevisiae BY4741 using rhodamine B staining. The treatment groups: (A) 0,5\% glucose, (B) 0 ppm, (C) $150 \mathrm{ppm}$, (D) $300 \mathrm{ppm}$, (E) $450 \mathrm{ppm}$, (F) $600 \mathrm{ppm}$ and (G) $750 \mathrm{ppm}$. Yeast cultured in YPD medium in low glucose $(0.5 \%)$ was used as positive control 


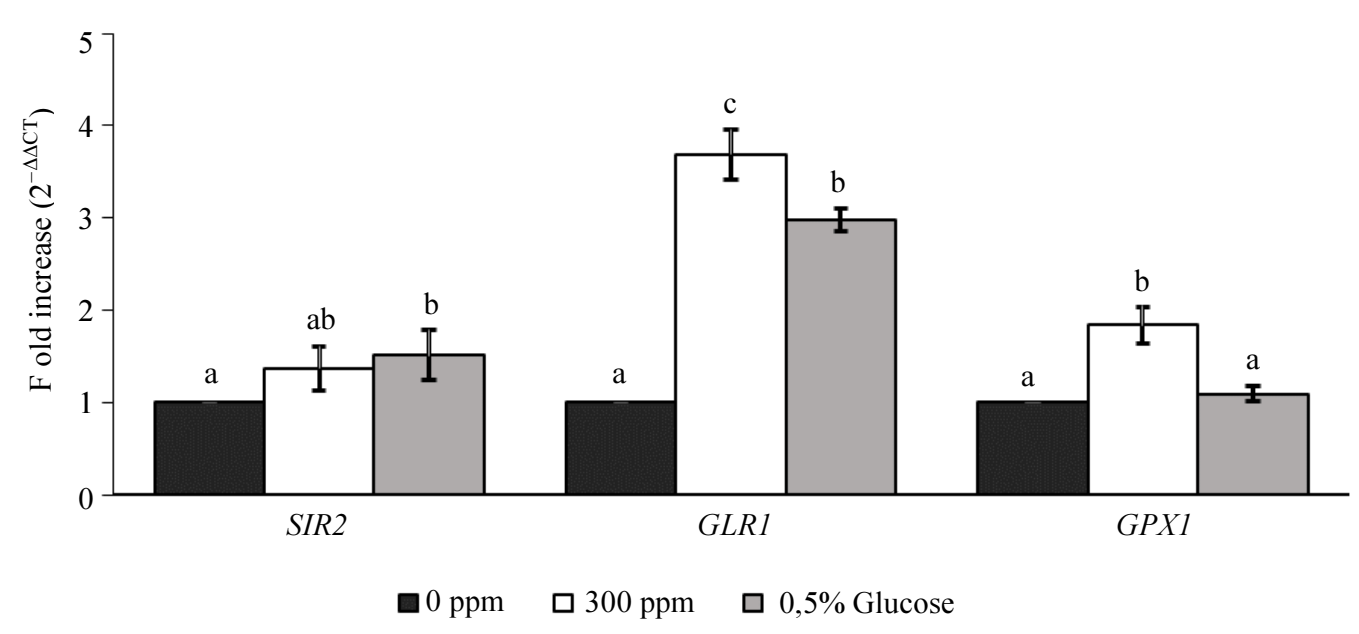

Fig. 5: The effects of Roselle petal extract on gene expression of aging pathway (SIR2) and tolerance against oxidative stress (GLR1 and $G P X 1$ ) investigated using RT-PCR experiment. Yeast cultured in $0.5 \%$ glucose was used as positive control. The level of gene expression SIR2, GLRI and GPX1 was normalized using reference gene ACT1. Bar data which followed with the same alphabet not significant in DMRT (Duncan's Multiple Range Test) 5\% test

\section{Real-Time PCR}

The RT-PCR analysis was performed to investigate the effects of roselle petal extract on gene expression that involves in response pathways against oxidative stress (GLR1 and GPX1) and aging pathways (SIR2). Treatment of extract $(300 \mathrm{ppm})$ upregulated the expression of all targeted genes, compared to that without extract treatment (Fig. 5). However, in comparison with glucose $0.5 \%$ (CR), the gene expression of extract treatment was much higher for gene expression involved in oxidative stress (GLR1 and GPX1) and lower for gene expression involved in the aging pathway (SIR2) (Fig. 5). CR condition could alter metabolic system of yeast, shifting from fermentation $(2 \%$ glucose) to respiration system. Activation of respiration system promoted formation of ROS and Styl MAP kinase. Consequently, the activated Styl gene could induce essential gene expression from stress response to extend yeast's lifespan. The level of those gene expressions at concentration of $300 \mathrm{ppm}$ indicated the involvement of the genes on antiaging and extract. The enhancing SIR2 expression would promote extension of yeast's lifespan (Lin et al., 2016), meanwhile the expression of GPXI and GLRI genes presented a mitochondrial enzymatic defense against ROS. GPX1 is recognized as one of the antioxidant enzymes that enable to alleviate free radicals and lipid peroxide (Shirazi et al., 2013), while GLR1 shows a pivotal role in protecting and avoiding cellular oxidative stress (Grant et al., 1996).

This present work is the first report that uncovers potential of roselle petal extract for inducing genes associated with response pathway against oxidative stress (GLR1 and GPXI) and aging pathway (SIR2). Previous report found that polyphenolic compound in apple extract could also upregulates gene expression of aging pathway (SIR2) and antioxidant (SOD1 and SOD2) in yeast (Xiang et al., 2011).

\section{Conclusion}

Antiaging and antioxidant properties of roselle petal extract were best found at $300 \mathrm{ppm}$. The extract was potential antioxidant agent since it enabled to induce intracellular yeast mechanisms against oxidative stress including mitochondrial activity and upregulation of key gene in aging pathway (SIR2) and genes involved in tolerance mechanism against oxidative stress (GPXI and $G L R 1)$. It is likely that the antiaging mechanism following extract treatments mimics to that calorie restriction conditions, in yeast cells.

\section{Acknowledgement}

This report is part of thesis of the first author, which is funded by Indonesia Endowment Fund for Education (LPDP) scholarship.

\section{Author's Contributions}

Sarima: Has contributed in a whole experiment, data analysis, paper writing and publication.

Rika Indri Astuti: Has handled data analysis, reviewed and revised the manuscript.

Anja Meryandini: Has reviewed and revised the manuscript.

\section{Ethics}

All authors read and approved the final version of this manuscript. There are not any ethical issues to declare that could arise after the publication of this manuscript. 


\section{References}

Alarcon-Aguilar, F.J., A. Zamilpa, M.D. Perez-Garcia, J.C. Almanza-Perez and E. RomeroNunez et al., 2007. Effect of Hibiscus sabdariffa on obesity in MSG mice. J. Ethnopharmacol., 114: 66-71.

DOI: $10.1016 /$ j.jep.2007.07.020

Al-Hashimi, A.G., 2012. Antioxidant and antibacterial activities of Hibiscus sabdariffa L extract. African J. Food Sci., 6: 506-511. DOI: 10.5897/AJFS12.099

Bai, K., X. Wen, Z. Jingfei, K. Tao and N. Yu et al., 2016. Assessment of free radical scavenging activity of dimethylglycine sodium salt and its role in providing protection against lipopolysaccharideinduced oxidative stress in mice. PLoS ONE, 11: 1-17. DOI: $0.1371 /$ journal.pone.0155393

Baracca, A., S. Gianluca, S. Giancarlo and L. Giorgio, 2003. Rhodamine 123 as a probe of mitochondrial membrane potential: Evaluation of proton flux through F0 during ATP synthesis. Biochim. Biophys. Acta, 1606: 137-146. DOI: $10.1016 / \mathrm{S} 0005-2728(03) 00110-5$

Burtner, R., C.J. Murakami, B.K. Kennedy and M. Kaeberlein, 2009. A molecular mechanism of chronological aging in yeast. Cell Cycle, 8: 1256-1270. DOI: $10.4161 /$ cc.8.8.8287

Carvajal-Zarrabal, O., P.M. Hayward-Jones, Z. OrtaFlores, C. Nolasco-Hipolito and D.M. BarradasDermitz et al., 2009. Effect of Hibiscus sabdariffa L. dried calyx ethanol extract on fat absorptionexcretion and body weight implication in rats. J. Biomed. Biotechnol., 2009: 394592-394592. DOI: $10.1155 / 2009 / 394592$

Da-Costa-Rocha, I., B. Bernd, S. Hartwig, P. Ivo and H. Michael, 2014. Hibiscus sabdariffa L. - a phytochemical and pharmacological review. Food Chem., 165: 424-443.

Dat, J., S. Vandenabeele, E. Vranova, M.M. Van and D. Inze et al., 2000. Dual action of the active oxygen species during plant stress responses. Cellular Molecular Life Sci., 57: 779-795.

DOI: $10.1007 / \mathrm{s} 000180050041$

Figueira, S.C.G., A.G. Carla, J.C. Maria, I.R. Ana and L.N. Malin et al., 2014. Antioxidant capacity, cytotoxicity and antimycobacterial activity of madeira archipelago endemic Helichrysum dietary and medicinal plants. Antioxidants, 3: 713-729. DOI: $10.3390 /$ antiox3040713

Floegel, A., O.K. Dae, J.C. Sang, I.K. Sung and K.C. Ock, 2011. Comparison of ABTS/DPPH assays to measure antioxidant capacity in popular antioxidant-rich US foods. J. Food Compos. Anal., 24: 1043-1048. DOI: 10.1016/J.JFCA.2011.01.008

Gladyshev, V.N., 2014. The free radical theory of aging is dead. Long live the damage theory. Antioxidants Redox Signal., 20: 727-731.

DOI: $10.1089 /$ ars.2013.5228
Grant, C.M., P.C. Lindsay, H.R. Jung and W.D. Ian, 1996. Yeast gluthation reductase is required for protection againtst oxidative stress and is a target gene for yAP 1 transcriptional regulation. Moleculer Microbiol., 21: 171-179. DOI: $10.1046 / \mathrm{j} .1365-2958.1996 .6351340 . \mathrm{x}$

Hayflick, L., 1976. The cell biology of human aging. New Engl. J. Med., 295: 1302-1308. DOI: 10.1056/NEJM197612022952308

Jarolim, S., J. Millen, G. Heeren, P. Laun and D.S. Goldfarb et al., 2004. A novel assay for replicative lifespan in Saccharomyces cerevisiae. FEMS Yeast Res., 5: 169-177. DOI: $10.1016 /$ j.femsyr.2004.06.015

Kaeberlein, M., 2010. Lessons on longevity from budding yeast. Nature, 464: 513-519. DOI: $10.1038 /$ nature 08981

Lin, W.L., Y.J. Hsieh, F.P. Chou, C.J. Wang and M.T. Cheng et al., 2003. Hibiscus protocatechuic acid inhibits lipopolysaccharide-induced rat hepatic damage. Arch. Toxicol., 77: 42-47. DOI: $10.1007 / \mathrm{s} 00204-002-0404-0$

Lin, Y., Y. Sun, Y. Weng, A. Matsuura and L. Xiang et al., 2016. Parishin from Gastropodia elata extends the lifespan of yeast via regulation of sir $2 / \mathrm{uth} 1 / \mathrm{TOR}$ signaling pathway. Oxidative Med. Cellular Longevity, 2016: 4074690-4074690. DOI: $10.1155 / 2016 / 4074690$

Liu, C.L., J.M. Wang, C.Y. Chu, M.T. Cheng and T.H. Tseng, 2002. In vivo protective effect of protocatechuic acid on tert-butyl hydroperoxideinduced rat hepatotoxicity. Food Chem. Toxicol., 40: 635-641. DOI: $10.1016 / \mathrm{S} 0278-6915(02) 00002-9$

Liu, K.S., S.M. Tsao and M.C. Yin, 2005. In vitro antibacterial activity of roselle calyx and protocatechuic acid. Phytotherapy Res., 19: 942-945. DOI: $10.1002 /$ ptr. 1760

Livak, K.J. and T.D. Schmittgen, 2001. Analysis of relative gene expression data using Real-Time quantitative PCR and the 2- $\Delta \Delta \mathrm{CT}$ method. Methods, 25: 402-408. DOI: $10.1006 /$ meth.2001.1262

Lu, Y., T.J. Khoo and C. Wiart, 2014. Antioxidant activity determination of citronellal and crude extracts of Cymbopogon citratus by 3 different methods. Pharmacol. Pharmacy, 5: 395-400. DOI: $10.4236 / p p .2014 .54047$

Maeta, K., N. Wataru, T. Yoshifumi, I. Shingo and I. Yoshiharu, 2007. Green tea polyphenols function as prooxidants to activate oxidative-stress-responsive transcription factors in yeasts. Applied Environ. Microbiol., 73: 572-580. DOI: 10.1128/AEM.01963-06

Marchi, E. and D. Cavalieri, 2008. Yeast as a model to investigate the mitochondrial role in adaptation to dietary fat and calorie surplus. Genes Nutrit., 3: 159-166. DOI: 10.1007/s12263-008-0101-6 
Mardiah, R.Z. Fransiska, P. Endang and D. Rizal, 2015. Anti-inflammatory of purple roselle extract in diabetic rats induced by Streptozotocin. Proc. Food Sci., 3: 182-189.

DOI: $10.1016 /$ j.profoo.2015.01.020

Niccoli, T. and P. Linda, 2012. Ageing as a risk factor for disease. Curr. Biol., 22: 1-12. DOI: 10.1016/j.cub.2012.07.024

Ochani, P.C. and P. D'Mello, 2009. Antioxidant and antihyperlipidemic activity of Hibiscus sabdariffa Linn. leaves and calyces extracts in rats. Indian J. Exp. Biol., 47: 276-282. PMID: 19382724

Re, R., N. Pellegrini, A. Proteggente, A. Pannala and M. Yang et al., 1999. Antioxidant activity applying an improved ABTS radical cation decolorization assay. Free Radical Biol. Med., 26: 1231-1237. DOI: $10.1016 / \mathrm{S} 0891-5849(98) 00315-3$

Roux, A.E., P. Chartrand, G. Ferbeyre and L.A. Rokeach, 2010. Fission yeast and other yeasts as emergent models to unravel cellular aging in eukaryotes. J. Gerontol., 65: 1-8. DOI: $10.1093 /$ gerona/glp 152

Salazar, A.R., L.A.P. Lopez, J.L. Arroyo, B.A. AlanGarza and N.W. Torres, 2009. Antimicrobial and antioxidant activities of plants from northeast of Mexico. Evidence-Based Complementary Alternative Med., 2011: 536139-536139. DOI: $10.1093 /$ ECAM/NEP127

Shirazi, A., M. Ehsan, G. Ghazale, M. Mehran and G. Mahmoud, 2013. Evaluation of radio-protective effect of melatonin on whole body irradiation induced liver tissue damage. Cell J., 14: 292-297. PMID: 23577309
Stephan, J., J. Franke and A.E. Ehrenhofer-Murray, 2013. Chemical genetic screen in fission yeast reveals roles for vacuolar acidification, mitochondrial fission and cellular GMP levels in lifespan extension. Ag. Cell, 12: 574-583. DOI: $10.1111 /$ acel.12077

Tseng, T.H., T.W. Kao, C.Y. Chu, F.P. Chou and W.L. Lin et al., 2000. Induction of apoptosis by hibiscus protocatechuic acid in human leukemia cells via reduction of retinoblastoma (RB) phosphorylation and Bcl-2 expression. Biochem. Pharmacol., 60: 307-315. DOI: 10.1016/S0006-2952(00)00322-1

Xiang, L., K. Sun, J. Lu, Y. Weng and A. Taoka et al., 2011. Anti-aging effects of phloridzin, an apple polyphenol, on yeast via SOD and SIR2 genes. Biosci. Biotechnol. Biochem., 75: 854-858. DOI: $10.1271 / \mathrm{bbb} .100774$

Young, A., 1997. Ageing and physiological functions. Philosophical Trans. Royal Society London, 352: 1837-43. DOI: 10.1098/rstb.1997.0169

Zuraida, E. Yerizel and E. Anas, 2015. Pengaruh pemberian ekstrak rosela (Hibiscus sabdariffa Linn) terhadap kadar malondialdehid dan aktivitas katalase tikus yang terpapar karbon tetraklorida. Andalas J. Health. 4: 795-802. 